\title{
Quantum-coherent phase oscillations in synchronization
}

\author{
Talitha Weiss, Stefan Walter, and Florian Marquardt \\ Friedrich-Alexander University Erlangen-Nürnberg (FAU), Department of Physics, Staudtstraße 7, 91058 Erlangen, Germany \\ and Max Planck Institute for the Science of Light, Staudtstraße 2, 91058 Erlangen, Germany
}

(Received 15 August 2016; published 11 April 2017)

\begin{abstract}
Recently, several studies have investigated synchronization in quantum-mechanical limit-cycle oscillators. However, the quantum nature of these systems remained partially hidden, since the dynamics of the oscillator's phase was overdamped and therefore incoherent. We show that there exist regimes of underdamped and even quantum-coherent phase motion, opening up new possibilities to study quantum synchronization dynamics. To this end, we investigate the Van der Pol oscillator (a paradigm for a self-oscillating system) synchronized to an external drive. We derive an effective quantum model which fully describes the regime of underdamped phase motion and additionally allows us to identify the quality of quantum coherence. Finally, we identify quantum limit cycles of the phase itself.
\end{abstract}

DOI: 10.1103/PhysRevA.95.041802

Introduction. Synchronization is commonly studied in so-called limit cycle (LC) oscillators that arise from an interplay of linear and nonlinear effects [1]. For instance, linear amplification causes an instability, whereas nonlinear damping limits the oscillator's dynamics to a finite amplitude. Notably, the phase remains free, which allows synchronization of the oscillator to an external periodic drive or other LC oscillators. A transition from the intrinsic LC motion toward synchronized oscillations occurs depending on the coupling strength to (and frequency mismatch of) the external reference.

Quantum synchronization, i.e., the study of quantum systems whose classical counterparts synchronize, has recently attracted increasing theoretical attention. So far, studies of quantum synchronization have only explored overdamped phase motion. This implies that the dynamics, although taking place in quantum systems, remains always incoherent and classical-like, ruling out the observation of interesting effects like quantum tunneling or superposition states of different synchronization phases. In the present Brief Report, we discover quantum-coherent phase dynamics.

Theoretical studies of quantum synchronization have been performed for different platforms, including optomechanics $[2,3]$, atoms and ions [4,5], Van der Pol (VdP) oscillators [6-10], and superconducting devices [11,12]. Measures of synchronization in the presence of quantum noise have been proposed in Refs. [5,13,14].

On the experimental side, only classical synchronization has been studied so far for a wide range of systems [15], including more recently optomechanical systems [16-19]. In the well-developed field of classical synchronization, overdamped phase motion is the standard ingredient both of phenomenological equations and microscopically derived models. For example, locking to an external force is described by the so-called Adler equation, a first-order differential equation for the phase. Similarly, synchronized optomechanical systems are described by the first-order phase equation of the Hopf-Kuramoto model [2,20,21]. However, it has been noticed that classical synchronization also allows for underdamped phase dynamics. For instance, the classical VdP oscillator features underdamped phase motion and even (synchronized) phase self-oscillations [1,22-24]. Both regimes have recently been observed experimentally using a nanoelectromechanical system [25]. Classical phase self-oscillations, also called phase trapping, have also been observed with coupled laser modes [26]. Furthermore, synchronized Josephson junction arrays can be mapped to the Kuramoto model including inertia [15,27].

Here we will show that a regime of quantum-coherent dynamics exists and that underdamped phase dynamics is a necessary but not sufficient condition to observe this regime. Rather, it is the dynamically generated nonequilibrium dephasing rate that has to become smaller than the oscillation frequency. Additionally, we identify phase self-oscillations in the quantum regime.

We obtain these insights for a paradigmatic model, the quantum version of the VdP oscillator subject to an external drive. The synchronization of the $\mathrm{VdP}$ oscillator to this external drive is an excellent test case to investigate universal synchronization behavior. We derive an effective quantum model that captures the regime of underdamped phase dynamics. This allows us to identify a quality factor for the quantum coherence. We illustrate the potentially long coherence times by showing that initial negativities of a Wigner density vanish slowly. Finally, we briefly discuss possible experimental realizations.

Quantum model. The quantum VdP oscillator subject to an external drive is described by the master equation $(\hbar=1)$

$$
\dot{\hat{\rho}}=-i\left[-\Delta \hat{b}^{\dagger} \hat{b}+i F\left(\hat{b}-\hat{b}^{\dagger}\right), \hat{\rho}\right]+\gamma_{1} \mathcal{D}\left[\hat{b}^{\dagger}\right] \hat{\rho}+\gamma_{2} \mathcal{D}\left[\hat{b}^{2}\right] \hat{\rho}
$$

with $\mathcal{D}[\hat{O}] \hat{\rho}=\hat{O} \hat{\rho} \hat{O}^{\dagger}-\left\{\hat{O}^{\dagger} \hat{O}, \hat{\rho}\right\} / 2$. Here, $\Delta=\omega_{d}-\omega_{0}$ is the detuning of the oscillator's natural frequency $\omega_{0}$ from the frequency of the external drive $\omega_{d}$ and $F$ is the driving force. The two dissipative terms in Eq. (1) describe gain and loss of one and two quanta at rates $\gamma_{1}$ and $\gamma_{2}$, respectively.

In Fig. 1 we show the steady-state Wigner function along with the corresponding phase probability distribution $P(\phi)=$ $\sum_{n, m=0}^{\infty} \frac{e^{i(m-n) \phi}}{2 \pi}\left\langle n\left|\hat{\rho}_{s S}\right| m\right\rangle$ [5] by numerically solving Eq. (1) for its steady state $\hat{\rho}_{s s}$. In the absence of an external force $(F=0)$, the two competing dissipation rates $\gamma_{1}$ and $\gamma_{2}$ lead to LC motion of the VdP oscillator, Fig. 1(a). For a finite applied force $(F \neq 0)$ and sufficiently small detuning $\Delta$, the $\mathrm{VdP}$ oscillator synchronizes to the external force and a fixed phase relation between the $\mathrm{VdP}$ oscillator and the force is present. 

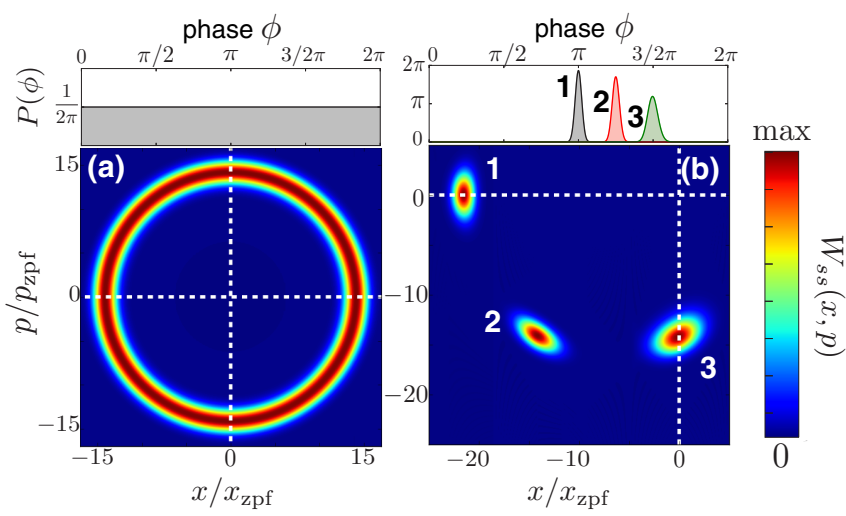

FIG. 1. Quantum synchronization. Steady-state Wigner density $W_{s s}(x, p)$ and phase probability distribution $P(\phi)$ of (a) an undriven $\left(F / \gamma_{1}=0\right)$ and (b) an externally driven $\left(F / \gamma_{1}=10\right) \mathrm{VdP}$ oscillator. (a) The ring-shaped Wigner function indicates LC motion. (b) With increasing detuning $\Delta / \gamma_{1}$, the synchronization phase changes and synchronization becomes weaker. Parameters: $\gamma_{2} / \gamma_{1}=5 \times 10^{-3}$, (a) $\Delta / \gamma_{1}=0$, (b) $1,2,3$ correspond to $\Delta / \gamma_{1}=0,0.5$, and 1 .

In the rotating frame, this corresponds to a localized Wigner density and a phase distribution $P(\phi)$ with a distinct peak. With increasing detuning, the $\mathrm{VdP}$ oscillator is less synchronized to the external force [related to the height and width of $P(\phi)$ ] and the synchronization phase [peak position of $P(\phi)$ ] is shifted [Fig. 1(b)].

These steady-state properties do not provide any information on the underlying synchronization dynamics, especially if we are trying to discover possible underdamped and quantumcoherent phase dynamics. To test for these regimes, we now derive an effective quantum model.

Effective quantum model. In the synchronized regime, the classical equation of motion for $\langle\hat{b}\rangle=\beta=R e^{i \phi}$,

$$
\dot{\beta}=i \Delta \beta+\frac{\gamma_{1}}{2} \beta-\gamma_{2}|\beta|^{2} \beta-F,
$$

has a stable fixed point $\beta_{s s}=R_{s s} e^{i \phi_{s s}}$. We linearize Eq. (1) around $\beta_{s s}$ by defining $\hat{b}=\beta_{s s}+\delta \hat{b}$, where $\delta \hat{b}$ describes fluctuations around $\beta_{s s}$. Neglecting terms of order $O\left(\delta \hat{b}^{3}\right)$ and higher, we obtain

$$
\dot{\hat{\rho}}_{\mathrm{eff}}=-i\left[\hat{H}_{\mathrm{eff}}, \hat{\rho}_{\mathrm{eff}}\right]+\gamma_{1} \mathcal{D}\left[\delta \hat{b}^{\dagger}\right] \hat{\rho}_{\mathrm{eff}}+4 \gamma_{2}\left|\beta_{s s}\right|^{2} \mathcal{D}[\delta \hat{b}] \hat{\rho}_{\mathrm{eff}},
$$

with the effective Hamiltonian

$$
\hat{H}_{\mathrm{eff}}=-\Delta \delta \hat{b}^{\dagger} \delta \hat{b}-i \frac{\gamma_{2}}{2}\left(\beta_{s s}^{2} \delta \hat{b}^{\dagger} \delta \hat{b}^{\dagger}-\beta_{s s}^{* 2} \delta \hat{b} \delta \hat{b}\right) .
$$

This effective model captures the major features of the full quantum model and thus allows at least qualitative predictions about the behavior of the system, while quantitative agreement varies with parameters. A comparison of the outcomes of Eqs. (1) and (3) can be found in the Supplemental Material [28]. The effective model is a squeezing Hamiltonian where the amount of squeezing depends on the classical steady-state solution $\beta_{s s}$.

Diagonalizing Eq. (4) leads to

$$
\hat{H}_{\text {diag }}=-\Omega_{\text {eff }} \hat{c}^{\dagger} \hat{c}+\text { const. }
$$
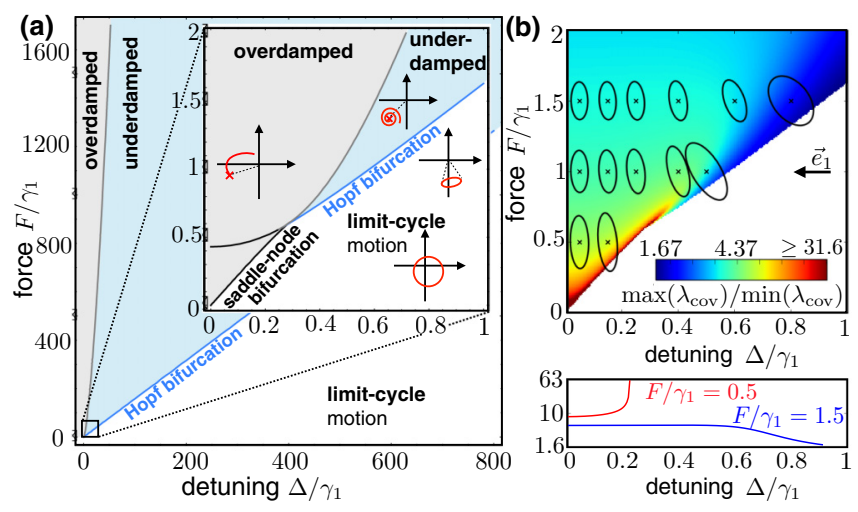

FIG. 2. Classical phase diagram and squeezing. (a) Overview of the classical synchronization regimes with sketches of typical phase-space trajectories. (b) Asymmetry of the steady-state squeezing ellipses, $\max \left(\lambda_{\text {cov }}\right) / \min \left(\lambda_{\text {cov }}\right)$, obtained from the effective model [30]. At the black crosses we show the squeezing ellipses (not to scale) with their radial direction aligned along $\vec{e}_{1}$. Two cuts at different forcing are shown below the figure. Parameters: $\gamma_{2} / \gamma_{1}=0.1$.

Here, $\delta \hat{b} e^{-i \theta / 2}=\cosh (\chi) \hat{c}+\sinh (\chi) \hat{c}^{\dagger}, A e^{i \theta}:=-i \gamma_{2} \beta_{s s}^{2} / 2$, $\tanh (2 \chi)=2 A / \Delta$, and $\Omega_{\text {eff }}=\sqrt{\Delta^{2}-4 A^{2}}$ is the effective frequency. The corresponding master equation reads

$$
\dot{\hat{\rho}}_{\text {diag }}=-i\left[\hat{H}_{\text {diag }}, \hat{\rho}_{\text {diag }}\right]+\Gamma_{\uparrow} \mathcal{D}\left[\hat{c}^{\dagger}\right] \hat{\rho}_{\text {diag }}+\Gamma_{\downarrow} \mathcal{D}[\hat{c}] \hat{\rho}_{\text {diag }},
$$

with $\Gamma_{\uparrow}=4 \gamma_{2}\left|\beta_{s s}\right|^{2} \sinh ^{2}(\chi)+\gamma_{1} \cosh ^{2}(\chi), \quad \Gamma_{\downarrow}=4 \gamma_{2}\left|\beta_{s s}\right|^{2}$ $\cosh ^{2}(\chi)+\gamma_{1} \sinh ^{2}(\chi)$, and neglecting fast rotating terms, such as $\hat{c} \hat{c} \hat{\rho}_{\text {eff }}$. The diagonalized, effective model is a damped harmonic oscillator with frequency $\Omega_{\text {eff }}$ and damping $\Gamma=$ $\Gamma_{\downarrow}-\Gamma_{\uparrow}$. This unambiguously allows us to identify an underdamped phase dynamics regime following the standard procedure for a harmonic oscillator; i.e., we require $\Delta^{2}>4 A^{2}$, which leads to a real-valued effective frequency $\Omega_{\text {eff }}$. This is consistent with the corresponding classical dynamics derived from Eq. (3), leading to a second-order differential equation of the phase,

$$
\delta \ddot{\phi}+\Gamma \delta \dot{\phi}+\Omega^{2} \delta \phi=0
$$

Here, $\delta \phi=\phi-\phi_{s s}$ describes phase deviations from the steady-state phase $\phi_{s s}$ and $\Omega=$ $\sqrt{\Delta^{2}+\left(\gamma_{2} R_{s s}^{2}-\gamma_{1} / 2\right)\left(3 \gamma_{2} R_{s s}^{2}-\gamma_{1} / 2\right)}$ is the bare frequency which is related to the effective frequency $\Omega_{\text {eff }}=$ $\sqrt{\Omega^{2}-\Gamma^{2} / 4}=\sqrt{\Delta^{2}-\gamma_{2}^{2}\left|\beta_{s s}\right|^{4}}$; cf. [25,28].

Before we discuss results from our effective quantum model, we briefly review the relevant features of the corresponding classical "phase diagram" of synchronization, Fig. 2(a). This phase diagram and its quantum analog will help us to identify the parameter regime of underdamped phase motion, where we then can check for quantum coherence. We obtain the boundaries between the regimes of the classical phase dynamics from a linear stability analysis of Eq. (2); cf. $[1,28]$. Notably, we distinguish two qualitatively different transitions from synchronization to no synchronization: At small forces, a saddle-node bifurcation characterizes the transition from synchronized (overdamped) dynamics directly 

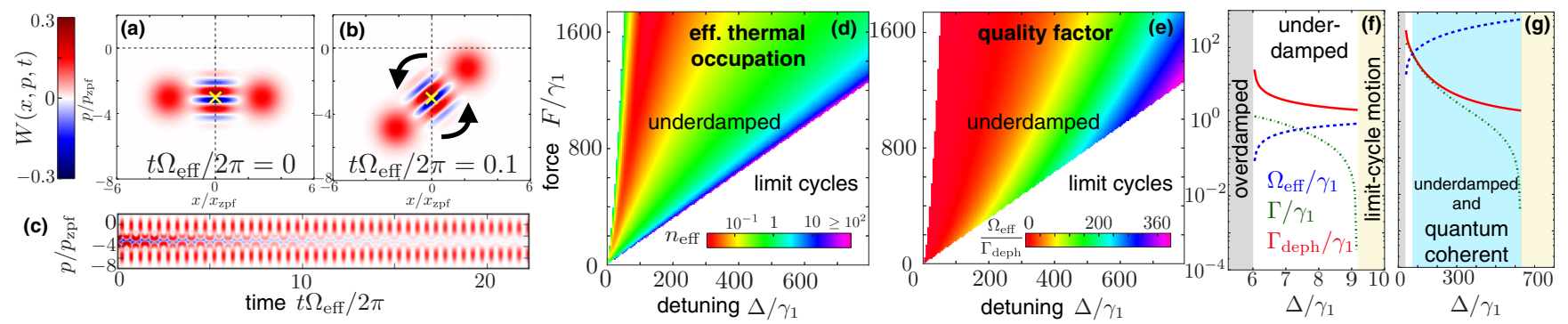

FIG. 3. Quantum coherence. Wigner densities $W(x, p, t)$ of (a) the initial state $|\Psi(t=0)\rangle \sim\left(\left|\beta_{s s}+2\right\rangle+\left|\beta_{s s}-2\right\rangle\right)$ and (b) at a later time. The underdamped phase dynamics rotates the state around the classical steady-state solution (yellow cross). (c) Wigner density $W(p, x=0, t)$ with negativities that remain visible for many oscillations. (d) Effective temperature in the underdamped regime, indicated by $n_{\text {eff }}$ The left white area corresponds to the overdamped regime. (e) Quality factor $\Omega_{\mathrm{eff}} / \Gamma_{\mathrm{deph}}$ in the underdamped regime. Panels (f) and (g) show the effective oscillation frequency $\Omega_{\text {eff }}$ (dashed blue), damping $\Gamma$ (dash-dotted green), and dephasing rate $\Gamma_{\text {deph }}$ (red) as a function of the detuning $\Delta$. (f) At small force $F / \gamma_{1}=1.5$ the dephasing remains the dominant rate. (g) In contrast, at larger force $F / \gamma_{1}=10^{3}$ the frequency $\Omega_{\text {eff }}$ can significantly exceed both the dephasing and the damping (quantum-coherent regime). Parameters: $\gamma_{2} / \gamma_{1}=0.1$, and (a) $-(\mathrm{c}) F / \gamma_{1}=1.5 \times 10^{3}$ and $\Delta / \gamma_{1}=7 \times 10^{2}$. Panels (a)-(c) show numerical solutions to the full model Eq. (1), while panels (d)-(g) show the rates obtained from our effective quantum model which characterize the behavior of the full system.

to the $\mathrm{LC}$ regime. At larger forces, a regime of underdamped phase motion opens up before a Hopf bifurcation marks the onset of a LC, which does not necessarily encircle the origin.

Since we are actually interested in a quantum regime, it is worthwhile to see that these two qualitatively different transitions have also important consequences for the quantum dynamics. In particular, we find a qualitative change of behavior in the squeezing properties of the steady state. Since $\hat{H}_{\text {eff }}$ is quadratic in $\delta \hat{b}$ the system is fully characterized by its covariance matrix $\sigma_{i j}=\operatorname{Tr}\left[\hat{\rho}_{\text {eff }}\left\{\hat{X}_{i}, \hat{X}_{j}\right\} / 2\right]$ with the quadratures $\hat{X}_{1}=\left(\delta \hat{b}+\delta \hat{b}^{\dagger}\right) / \sqrt{2}$ and $\hat{X}_{2}=-i\left(\delta \hat{b}-\delta \hat{b}^{\dagger}\right) / \sqrt{2}$. The eigenvalues $\lambda_{\text {cov }}$ of the covariance matrix determine the shape of the squeezing ellipse [29]. Their ratio (the asymmetry of the ellipses) is shown in Fig. 2(b). Notably, at small forces, it increases with larger detuning. In contrast, at larger forces where we predict underdamped phase dynamics, the ellipses become more circular while increasing the detuning $\Delta$. Thus, the squeezing behavior can be used as an indicator for the existence of a quantum regime of underdamped phase motion. The effective model becomes unstable if $\Gamma=0$, which corresponds to the classical fixed point losing its stability. Additional details on squeezing can be found in the Supplemental Material [28].

Quantum coherence. Studying the effective model, we have identified the quantum regime of underdamped phase motion. Now we demonstrate that within this regime, it is possible to preserve quantum coherence for a significant time. To this end, we choose an initial state which possesses negativities in its Wigner function, Fig. 3(a), and show that these negativities persist for a long time compared to the characteristic time scale of the dynamics $\Omega_{\text {eff }}^{-1}$. The dynamics due to Eq. (1) leads to a rotation of the state around the classical steady state $\beta_{s s}$, Fig. 3(b). Notably, this dynamical evolution has little influence on the coherence and the negativities of the Wigner density survive many oscillations of the system [Fig. 3(c)]. After the loss of coherence, the state remains in a classical mixture of two displaced states and settles into the steady state only on an even longer timescale; see Ref. [28] for a complete overview. All Wigner densities in Figs. 3(a)-3(c) are obtained by numerically solving the full master equation (1).

This behavior is successfully predicted by our effective model, which allows us to quantify quantum coherence within the underdamped regime and eventually identify a quantumcoherent regime. The time scale on which the quantum system approaches the steady state is approximately given by the damping $\Gamma$. Thus, a necessary condition to observe quantumcoherent motion is $\Omega_{\mathrm{eff}}>\Gamma$. Approaching the classical Hopf bifurcation, the damping $\Gamma$ becomes arbitrarily small. However, a small damping does not imply a small dephasing rate $\Gamma_{\text {deph }}=\Gamma_{\uparrow}+\Gamma_{\downarrow}$. The dephasing rate ultimately determines the lifetime of negativities, i.e., quantum coherence. With $\Gamma_{\uparrow}=\Gamma n_{\text {eff }}$ and $\Gamma_{\downarrow}=\Gamma\left(n_{\text {eff }}+1\right)$, the dephasing rate $\Gamma_{\text {deph }}$ depends on both the damping $\Gamma$ and the effective occupation of the VdP oscillator $n_{\text {eff. This effective occupation comes }}$ about due to the driven-dissipative character of the quantum oscillator even at zero environmental temperature, also called quantum heating [31]. It increases towards the boundaries of the underdamped regime, Fig. 3(d), counteracting the decreasing damping. Additional insight is obtained by identifying a quality factor for quantum coherence, $\Omega_{\mathrm{eff}} / \Gamma_{\mathrm{deph}}$, which determines the lifetime of negativities in the Wigner density. Close to the instability and, more importantly, at large forcing and detuning, $\Omega_{\mathrm{eff}} / \Gamma_{\text {deph }}$ increases and can become significantly larger than 1 [Fig. 3(e)]. This is the quantumcoherent regime where negativities of the Wigner density can survive many oscillations of the system [Fig. 3(c)]. Regarding Fig. 3(e), the only remaining dimensionless parameter (apart from the normalized force and detuning) is the ratio of the damping rates $\gamma_{2} / \gamma_{1}$. It influences the region of stability of the effective model. For instance, increasing $\gamma_{2} / \gamma_{1}$ shifts the instability $(\Gamma=0)$ to larger detuning. This allows to achieve a comparable quality factor $\Omega_{\mathrm{eff}} / \Gamma_{\mathrm{deph}}$ at smaller forcing but similar detuning-mainly because $\Omega_{\text {eff }}$ increases with $\Delta$. In Figs. 3(f) and 3(g) we show all relevant rates in the underdamped regime at small and large forcings, respectively. In both cases $\Omega_{\text {eff }}$ increases, while $\Gamma$ and $\Gamma_{\text {deph }}$ decrease with larger detuning. At small force [Fig. 3(f)], the dephasing rate remains the largest rate in the entire underdamped regime. 


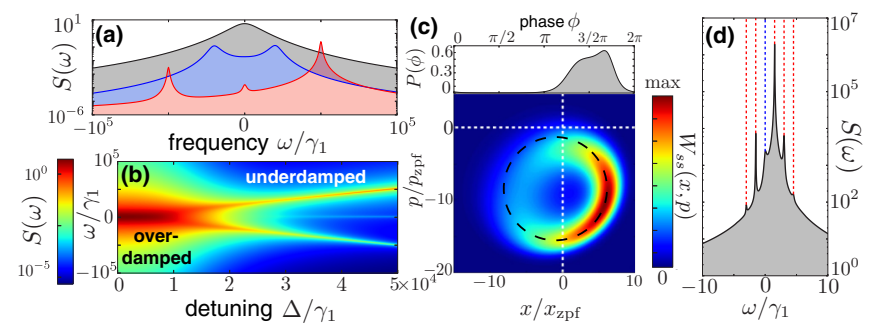

FIG. 4. Spectrum. Panels (a) and (b) show the spectrum $S(\omega)$ of a synchronized VdP oscillator for different detunings $\Delta / \gamma_{1}=0$ (upper black spectrum), $\Delta / \gamma_{1}=2 \times 10^{4}$ (middle blue spectrum), and $\Delta / \gamma_{1}=5 \times 10^{4}$ (lower red spectrum). In the overdamped regime (upper black) the spectrum shows a single peak at $\omega=0$, while in the underdamped regime (middle blue and lower red) double peaks at $\pm \Omega_{\text {eff }}$ emerge. Parameters: $\gamma_{2} / \gamma_{1}=2 \times 10^{3}, F / \gamma_{1}=2 \times 10^{4}$. (c) Steady-state Wigner density $W_{s s}(x, p)$ and phase probability distribution $P(\phi)$ of a VdP oscillator showing phase self-oscillations, i.e., a ring-like Wigner density not encircling the origin (indicated by the dashed black line). The corresponding spectrum (d) shows multiple peaks at higher harmonics. Parameters for panels (c) and (d): $\gamma_{2} / \gamma_{1}=5 \times 10^{-3}, F / \gamma_{1}=10, \Delta / \gamma_{1}=1.55$. All subfigures (a)(d) show numerical solutions of the full model Eq. (1).

Notably, for large $F$ [Fig. 3(g)], we find that $\Omega_{\text {eff }}$ can become significantly larger than both $\Gamma$ and $\Gamma_{\text {deph }}$, thus entering the quantum-coherent regime. This is the key element to observing long-lived quantum coherence.

Spectrum. To shed more light on the possibility to experimentally observe the transition from overdamped to underdamped synchronization dynamics, we investigate the spectrum $S(\omega)=\int_{-\infty}^{\infty} d t e^{i \omega t}\left\langle\hat{b}^{\dagger}(t) \hat{b}(0)\right\rangle$. We obtain $S(\omega)$ from the steady state of Eq. (1) by applying the quantum regression theorem or analytically from the effective model; see Supplemental Material [28]. The spectrum carries information on the frequencies of the driven VdP oscillator. Figure 4(a) shows $S(\omega)$ for a fixed external force and various detunings, corresponding to the overdamped (upper black spectrum) and underdamped (middle blue, lower red spectra) regime. In the overdamped regime the spectrum shows a single peak close to $\omega=0$, indicating synchronization to the external force. With increasing detuning, the spectrum develops from a single peak to two peaks which now sit at approximately $\pm \Omega_{\text {eff. }}$ A small remainder of the central peak at $\omega=0$ becomes visible for a larger splitting of the main peaks. The emerging double peaks clearly indicate the transition from overdamped to underdamped phase dynamics [Fig. 4(b)]. The increasing asymmetry of $S(\omega)$ results from the coupling of amplitude and phase dynamics. For even larger detuning, synchronization is lost which ultimately leads to a single peak in the spectrum at $\omega=\Delta$. A recent experiment synchronized two nanomechanical oscillators by coupling to a common cavity mode [17]. Curiously, the cavity output spectrum showed sidebands next to the common frequency of the locked oscillators. These sidebands were suggested to arise from (classical) underdamped phase motion of the oscillators, which is also consistent with the classical limit of our theory.

Interestingly, we find that the phase can even undergo self-oscillations. In the quantum regime, these phase self- oscillations appear (in analogy to the classical scenario) at the boundary of underdamped phase motion just before the loss of synchronization occurs. A circular LC opens up around the former stable fixed point. In the quantum regime this is smeared by quantum fluctuations and becomes visible only once the LC is large enough. If that LC expands even further, it will eventually come to resemble the original unsynchronized state: The LC encircles the origin of phase space and the corresponding phase distribution is flat [Fig. 1(a)]. However, in Fig. 4(c), this is not yet the case, i.e., the LC does not encircle the origin. The oscillator has still a tendency to be locked to the phase of the external force. This is also reflected in the corresponding phase distribution $P(\phi)$ which becomes asymmetric and shows the onset of a double peak structure. Notably, phase self-oscillations are accompanied by the appearance of a series of peaks in the spectrum [Fig. 4(d)], representing higher harmonics of the main phase-oscillation frequency.

Experimental realization. The regime of underdamped quantum phase motion and even quantum phase selfoscillations could be experimentally studied in a variety of systems. For instance, trapped ions are promising candidate systems for studying synchronization in the quantum regime $[6,8]$. The possibility to prepare nonclassical states experimentally [32] allows for probing the quantum-coherent nature of the underdamped phase dynamics. Based on parameters for trapped ${ }^{171} \mathrm{Yb}^{+}$ions $[6,33,34]$, we estimate that it should be possible to observe significant quantum coherence. In this scenario, the negative and nonlinear damping are both of the order of $\mathrm{kHz}$, with $\gamma_{2} / \gamma_{1} \sim 1$. To observe quantum-coherent underdamepd phase dynamics the detuning $\Delta$ and the external force $F$ should be a few hundred $\mathrm{kHz}$ each. This is realistic, with frequencies of the motional state in the $\mathrm{MHz}$ regime. Furthermore, mechanical self-oscillations in cavity optomechanics have been discussed theoretically [35] and observed experimentally $[36,37]$. Thus, they are well suited to study synchronization, and classical synchronization phenomena have already been demonstrated experimentally [16-19]. Yet another possible platform to observe quantum-coherent phase motion are superconducting microwave circuits. These are exceptional and highly tuneable platforms for experimentally investigating quantum systems. In principle, arbitrary quantum states can be realized [38-40]. Even, the faithful engineering of two-photon losses in such systems has been demonstrated [41]. This makes them very interesting for studying quantumcoherent phase motion and phase self-oscillations of a quantum $\mathrm{VdP}$ oscillator.

Conclusion. We have shown that the phase of a synchronized quantum Van der Pol oscillator exhibits intriguing underdamped and even quantum-coherent phase dynamics around the synchronized steady state. In order to explore this interesting regime, we have developed an effective quantum model and identified where the dephasing rate becomes sufficiently small to observe quantum-coherent phase motion. As a direct consequence, we have shown that this preserves a nonclassical quantum state for many phase oscillations. We estimate that this could readily be observed in state-ofthe-art experiments. While we have analyzed the simplest synchronization phenomenon, to an external drive, the regime identified here will also show up in the quantum phase 
dynamics of two coupled oscillators or even lattices [2]. In the latter case, phenomena such as quantum motion of phase vortices may potentially become observable.
Acknowledgments. We acknowledge financial support by the Marie Curie ITN cQOM and the ERC OPTOMECH.
[1] J. Kurths, A. Pikovsky, and M. Rosenblum, Synchronization: A Universal Concept in Nonlinear Sciences (Cambridge University Press, Cambridge, UK, 2001).

[2] M. Ludwig and F. Marquardt, Phys. Rev. Lett. 111, 073603 (2013).

[3] T. Weiss, A. Kronwald, and F. Marquardt, New J. Phys. 18, 013043 (2016).

[4] M. Xu, D. A. Tieri, E. C. Fine, J. K. Thompson, and M. J. Holland, Phys. Rev. Lett. 113, 154101 (2014).

[5] M. R. Hush, W. Li, S. Genway, I. Lesanovsky, and A. D. Armour, Phys. Rev. A 91, 061401 (2015).

[6] T. E. Lee and H. R. Sadeghpour, Phys. Rev. Lett. 111, 234101 (2013).

[7] S. Walter, A. Nunnenkamp, and C. Bruder, Phys. Rev. Lett. 112, 094102 (2014).

[8] T. E. Lee, C. K. Chan, and S. Wang, Phys. Rev. E 89, 022913 (2014).

[9] S. Walter, A. Nunnenkamp, and C. Bruder, Ann. Phys. (Berlin) 527, 131 (2014).

[10] N. Lörch, E. Amitai, A. Nunnenkamp, and C. Bruder, Phys. Rev. Lett. 117, 073601 (2016).

[11] V. M. Vinokur, T. I. Baturina, M. V. Fistul, A. Y. Mironov, M. R. Baklanov, and C. Strunk, Nature (London) 452, 613 (2008).

[12] A. M. Hriscu and Y. V. Nazarov, Phys. Rev. Lett. 110, 097002 (2013).

[13] A. Mari, A. Farace, N. Didier, V. Giovannetti, and R. Fazio, Phys. Rev. Lett. 111, 103605 (2013).

[14] V. Ameri, M. Eghbali-Arani, A. Mari, A. Farace, F. Kheirandish, V. Giovannetti, and R. Fazio, Phys. Rev. A 91, 012301 (2015).

[15] J. A. Acebrón, L. L. Bonilla, C. J. Pérez Vicente, F. Ritort, and R. Spigler, Rev. Mod. Phys. 77, 137 (2005).

[16] M. Zhang, G. S. Wiederhecker, S. Manipatruni, A. Barnard, P. McEuen, and M. Lipson, Phys. Rev. Lett. 109, 233906 (2012).

[17] M. Bagheri, M. Poot, L. Fan, F. Marquardt, and H. X. Tang, Phys. Rev. Lett. 111, 213902 (2013).

[18] K. Shlomi, D. Yuvaraj, I. Baskin, O. Suchoi, R. Winik, and E. Buks, Phys. Rev. E 91, 032910 (2015).

[19] M. Zhang, S. Shah, J. Cardenas, and M. Lipson, Phys. Rev. Lett. 115, 163902 (2015).

[20] G. Heinrich, M. Ludwig, J. Qian, B. Kubala, and F. Marquardt, Phys. Rev. Lett. 107, 043603 (2011).

[21] R. Lauter, C. Brendel, S. J. M. Habraken, and F. Marquardt, Phys. Rev. E 92, 012902 (2015).

[22] T. Chakraborty and R. H. Rand, Int. J. Nonlinear Mech. 23, 369 (1988).

[23] D. G. Aronson, G. B. Ermentrout, and N. Kopell, Phys. D (Amsterdam, Neth.) 41, 403 (1990).
[24] A. Pikovsky, M. Rosenblum, and J. Kurths, Int. J. Bifurcation Chaos 10, 2291 (2000).

[25] T. Barois, S. Perisanu, P. Vincent, S. T. Purcel, and A. Ayari, New J. Phys. 16, 083009 (2014).

[26] J. Thévenin, M. Romanelli, M. Vallet, M. Brunel, and T. Erneux, Phys. Rev. Lett. 107, 104101 (2011).

[27] B. R. Trees, V. Saranathan, and D. Stroud, Phys. Rev. E 71, 016215 (2005).

[28] See Supplemental Material at http://link.aps.org/supplemental/ 10.1103/PhysRevA.95.041802 for additional details about classical synchronization, squeezing, the spectra, and a comparison between the full an effective model.

[29] D. Walls and G. J. Milburn, Quantum Optics (Springer-Verlag, Berlin, 2008).

[30] Quantum noise leads to a finite threshold for synchronization $[2,7]$. Below this threshold, the effective model is not applicable since classically a stable fixed point exists, but the quantum system settles on a LC. To improve the readability of Fig. 2(b) we nevertheless also plot the region where the effective model fails which here is only the case for very small force and detuning.

[31] M. I. Dykman, M. Marthaler, and V. Peano, Phys. Rev. A 83, 052115 (2011).

[32] D. Leibfried, D. M. Meekhof, B. E. King, C. Monroe, W. M. Itano, and D. J. Wineland, Phys. Rev. Lett. 77, 4281 (1996).

[33] K. R. Islam, Ph.D. thesis, University of Maryland, College Park, MD, 2012, http://drum.lib.umd.edu/handle/1903/13218.

[34] D. Leibfried, R. Blatt, C. Monroe, and D. Wineland, Rev. Mod. Phys. 75, 281 (2003).

[35] F. Marquardt, J. G. E. Harris, and S. M. Girvin, Phys. Rev. Lett. 96, 103901 (2006).

[36] T. J. Kippenberg, H. Rokhsari, T. Carmon, A. Scherer, and K. J. Vahala, Phys. Rev. Lett. 95, 033901 (2005).

[37] C. Metzger, M. Ludwig, C. Neuenhahn, A. Ortlieb, I. Favero, K. Karrai, and F. Marquardt, Phys. Rev. Lett. 101, 133903 (2008).

[38] M. Hofheinz, E. M. Weig, M. Ansmann, R. C. Bialczak, E. Lucero, M. Neeley, A. D. O'Connell, H. Wang, J. M. Martinis, and A. N. Cleland, Nature (London) 454, 310 (2008).

[39] S. Deleglise, I. Dotsenko, C. Sayrin, J. Bernu, M. Brune, J. M. Raimond, and S. Haroche, Nature (London) 455, 510 (2008).

[40] M. Hofheinz, H. Wang, M. Ansmann, R. C. Bialczak, E. Lucero, M. Neeley, A. D. O'Connell, D. Sank, J. Wenner, J. M. Martinis, and A. N. Cleland, Nature (London) 459, 546 (2009).

[41] Z. Leghtas, S. Touzard, I. M. Pop, A. Kou, B. Vlastakis, A. Petrenko, K. M. Sliwa, A. Narla, S. Shankar, M. J. Hatridge, M. Reagor, L. Frunzio, R. J. Schoelkopf, M. Mirrahimi, and M. H. Devoret, Science 347, 853 (2015). 